\title{
Topological orders competing for the Dirac surface state in FeSeTe surfaces
}

\author{
Xianxin Wu, ${ }^{1,2}$ Suk Bum Chung $\odot,{ }^{3,4,5}$ Chaoxing Liu, ${ }^{1}$ and Eun-Ah Kim $\odot^{6, *}$ \\ ${ }^{1}$ Department of Physics, the Pennsylvania State University, University Park, Pennsylvania 16802, USA \\ ${ }^{2}$ Beijing National Laboratory for Condensed Matter Physics, and Institute of Physics, Chinese Academy of Sciences, Beijing 100190, China \\ ${ }^{3}$ Department of Physics, University of Seoul, Seoul 02504, Korea \\ ${ }^{4}$ Natural Science Research Institute, University of Seoul, Seoul 02504, Korea \\ ${ }^{5}$ School of Physics, Korea Institute for Advanced Study, Seoul 02455, Korea \\ ${ }^{6}$ Department of Physics, Cornell University, Ithaca, New York 14853, USA
}

(Received 17 June 2020; revised 20 November 2020; accepted 18 December 2020; published 21 January 2021)

FeSeTe has recently emerged as a leading candidate material for the two-dimensional topological superconductivity (TSC). Two reasons for the excitement are the high $T_{c}$ of the system and the fact that the Majorana zero modes (MZMs) inside the vortex cores live on the exposed surface rather than at the interface of a heterostructure as in the proximitized topological insulators. However, the recent scanning tunneling spectroscopy data have shown that, contrary to the theoretical expectation, the MZM does not exist inside every vortex core. Hence there are "full" vortices with MZMs and "empty" vortices without MZMs. Moreover, the fraction of "empty" vortices increases with an increase in the magnetic field. We propose the possibility of two distinct gapped states competing for the topological surface states in FeSeTe: the TSC and half quantum anomalous Hall (hQAH). The latter is promoted by a magnetic field through the alignment of impurity magnetic moments such as $\mathrm{Fe}$ interstitials. When hQAH takes over the topological surface state, the surface will become transparent to scanning tunneling microscopy and the nature of the vortices in such regions will appear identical to what is expected of the vortices in the bulk, i.e., empty. The unmistakable signature of the proposed mechanism for empty vortices will be the existance of chiral Majorana modes(CMM) at the domain wall between an hQAH region and a TSC region. Such CMMs should be observable by measuring local density of states along a line connecting an empty vortex to a nearby full vortex.

DOI: 10.1103/PhysRevResearch.3.013066

\section{INTRODUCTION}

One particularly exciting feature of the topological insulator (TI) is its potential to host the Majorana zero mode (MZM), which has led to many proposals [1-4] and attempts [5-9] to realize MZM through introducing superconducting pairing to the TI surface state. Early works focused on inducing topological superconductivity (TSC) through the proximity effect $[3,10-12]$. More recently, the prospect of FeSeTe, possessing at its surface the equivalent of TI surface states with superconducting gaps induced by proximity to the intrinsic high $T_{c}$ bulk superconductivity, raised much enthusiasm [13-16]. More recently it has been recognized that such a state possesses a higher-order topology [17-21].

Intensive experimental investigations of FeSeTe confirmed the existence of a Dirac surface state in the normal state above $T_{c}$ [22]. The predicted evidence for the MZM in the vortex core in the superconducting state was a zero-bias

\footnotetext{
*eun-ah.kim@cornell.edu

Published by the American Physical Society under the terms of the Creative Commons Attribution 4.0 International license. Further distribution of this work must maintain attribution to the author(s) and the published article's title, journal citation, and DOI.
}

peak in scanning tunneling microscopy (STM). Indeed, STM is a particularly suitable probe for the MZM in this material as it would exist at the surface [11,12]. Despite several observations of zero-bias peaks in cores of some vortices [23-26], an apparent contradiction to the prediction has also been observed in the increasing fraction of "empty" vortices without a zero-bias peak upon the increase in magnetic field $[27,28]$. A careful study [27] revealed that the "empty" vortices cannot be accounted for by a simple picture of pair-wise annihilation of MZM between two near-by vortices. Although Ref. [29] showed that a model allowing for long-range interactions among far-separated MZMs can, in principle, explain the "empty" vortices, an alternative explanation with simpler starting point and a falsifiable prediction is desirable.

Here we provide an alternative interpretation of the observed "empty" vortices based on the role of the magnetic field in aligning local moments of $\mathrm{Fe}$ interstitials. Our main physical picture is summarized in Figs. 1(a)-1(d). As it is known from the study of magnetic dopants added to TI surface states, the exchange field from magnetic impurities also gaps out the TI surface state to form the half quantum anomalous Hall (hQAH) state with half-integer quantization of Hall conductivity [2,30-32] [Figs. 1(a)-1(b)]. Uneven distribution of $\mathrm{Fe}$ interstitials can nucleate the hQAH regions on the surface of $\mathrm{FeSeTe}$ when their moments get aligned with external magnetic fields [Fig. 1(c)], preventing TSC to form in that 
(a)

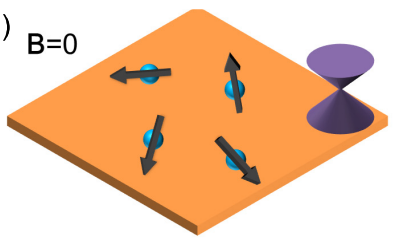

(c)

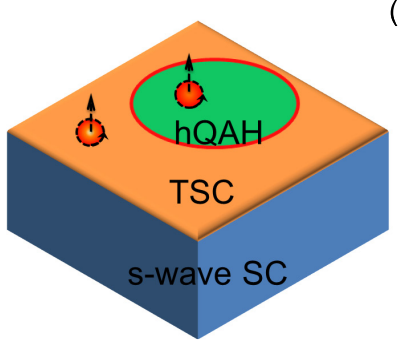

(b)

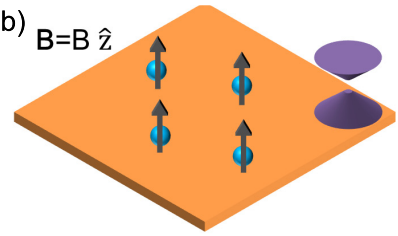

(d)

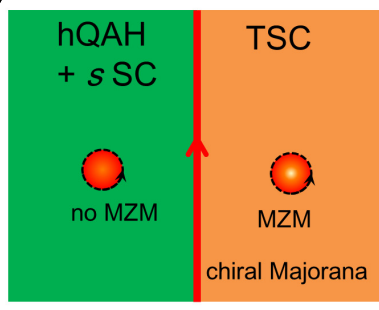

FIG. 1. (a) Gapless Dirac surface state with random magnetic moments of Fe interstitials and (b) the gaped surface state when magnetic moments are aligned by external magnetic fields. (c) Domain wall between the TSC region (with dominant superconducting gap) and the hQAH region (with dominant magnetic gap) on the surface of FeTeSe. (d) MZM exists at the vortex core (the red spots) in the TSC region, but not in the hQAH region. The CMM exists at the boundary between the TSC and hQAH regions.

very region. Such a hQAH surface state will reveal the bulk superconductivity to STM and the vortices penetrating hQAH surface will show properties of the bulk superconducting state with the topologically trivial $s_{ \pm}$pairing $[33,34]$, i.e., becoming "empty". With increasing magnetic fields, more hQAH regions are nucleated on the surface of $\mathrm{FeSeTe}$, thus providing a natural explanation of the increasing faction of empty vortices observed in experiments. Interestingly, it has been known that a boundary between hQAH and TSC should host a chiral Majorana mode (CMM) [4,35-37]. Hence our key prediction is that the MZM that would have been in the vortex core transforms into the CMM located at the boundary between the hQAH and TSC on the surface of FeTeSe [Fig. 1(d)]. In the rest of this article, we first present our proposal using a lowenergy effective theory and then support it with a numerical simulation based on a microscopic model in FeSeTe.

\section{EXCHANGE FIELD AND LOW-ENERGY EFFECTIVE THEORY}

Due to the topologically nontrivial properties in FeSeTe, there are topological Dirac surface states. As noted by neutron scattering experiments [38], and by Jiang et al. [39], the interstitial $\mathrm{Fe}$ atoms can provide magnetic impurities and generate magnetic domains in FeSeTe. Although the impurity moments will point in random directions at zero field [Fig. 1(a)], the external field applied to create vortices would align the impurity moments [Fig. 1(b)]. In the regions with a higher concentration of aligned impurity moments, the exchange field generated by these moments would couple to the topological surface state as in magnetically doped TIs [40-46]. Such an exchange coupling can be captured by $H_{e x}(\mathbf{r})=-\mathcal{J}_{0} \sum_{i} \mathbf{S}_{i}$. $\mathbf{s} \delta\left(\mathbf{r}-\mathbf{r}_{i}\right)$, where $\mathbf{s}=\frac{\hbar}{2} \sigma$ is the surface state electron spin, $\mathbf{S}_{i}$ and $\mathbf{r}_{i}$ are the spin and location, respectively, of the Fe interstitial and $\mathcal{J}_{0}$ is the coupling constant. This exchange

field will be heterogeneous depending on the distribution of the interstitials. We consider the mean field approximation for the exchange field, leading to the form $H_{e x}(\mathbf{r})=-\mathbf{I}_{\mathbf{e x}}(\mathbf{r}) \cdot \sigma$, where $\mathbf{I}_{\mathbf{e x}}(\mathbf{r})=\frac{\mathcal{J}_{0} \hbar}{2} \sum_{i}\left\langle\mathbf{S}_{i} \delta\left(\mathbf{r}-\mathbf{r}_{i}\right)\right\rangle_{\text {local }}$ is a smoothly varying field with $\langle\ldots\rangle_{\text {local }}$ representing the average over a small region for Fe moments. In an ordinary topological insulator, such a heterogeneous exchange field should result in the hQAH effect with spatially varying gaps for the Dirac surface state [47]. However, nontopological bands crossing the Fermi level will mask hQAH states in the normal state of FeSeTe.

Once the system develops superconductivity, the hQAH and TSC can compete as the two distinct ways of gapping the Dirac surface state. Moreover, the hQAH region will reveal itself by leaving the bulk superconductivity bare when the exchange gap dominates over the superconducting gap. This can be captured by the Bogoliubov-de Gennes (BdG) Hamiltonian for the Dirac surface state with both the exchange field and the $s$-wave pairing in the basis $\left(c_{\mathbf{k}, \uparrow}, c_{\mathbf{k}, \downarrow}, c_{-\mathbf{k}, \downarrow}^{\dagger},-c_{-\mathbf{k}, \uparrow}^{\dagger}\right)^{T}:$

$$
\mathcal{H}_{\mathrm{BdG}}=(v \mathbf{k} \cdot \sigma-\mu) \tau_{z}-I_{e x} \sigma_{z}+\Delta \tau_{x},
$$

where $\sigma_{i}$ and $\tau_{i}$ are the Pauli matrices in the spin space and particle-hole space, respectively. Here we assumed an $s$-wave gap to be real and only consider an exchange field along the $z$ direction; possible change in the pairing gap symmetry due to the exchange field [46] was not included as the exchange field has little effect on the bulk pairing. It is straightforward to find upon increase in the exchange term that the superconducting gap for BdG quasiparticles closes at a critical exchange field strength of [48-50]

$$
I_{e x, c}^{2}=|\Delta|^{2}+\mu^{2} .
$$

When $\left|I_{e x}\right|<\left|I_{e x, c}\right|$, the TSC dominates to support vortex core MZMs, which can be explicitly obtained by choosing $\Delta \tau_{x} \rightarrow|\Delta|\left(\tau_{x} \cos \theta-\tau_{y} \sin \theta\right)$ substitution $(\theta$ is the azimuthal angle) with a superconducting vortex at the origin. The zero-energy mode we obtain for $\left|I_{e x}\right|<|\mu|$ reads [3]

$$
\left[\begin{array}{c}
\psi_{\uparrow}(\mathbf{r}) \\
\psi_{\downarrow}(\mathbf{r}) \\
\psi_{\downarrow}^{\dagger}(\mathbf{r}) \\
-\psi_{\uparrow}^{\dagger}(\mathbf{r})
\end{array}\right]=\frac{e^{-\int_{0}^{r} d r^{\prime} \frac{\Delta \Delta}{\hbar v}}}{\left(\mu^{2}-I_{e x}^{2}\right)^{\frac{1}{4}}}\left[\begin{array}{c}
e^{-i \frac{\pi}{4}} \sqrt{\mu+I_{e x}} J_{0}\left(\frac{\sqrt{\mu^{2}-I_{e x}^{2}}}{\hbar v} r\right) \\
e^{i \frac{\pi}{4}} e^{i \theta} \sqrt{\mu-I_{e x}} J_{1}\left(\frac{\sqrt{\mu^{2}-I_{e x}^{2}}}{\hbar v} r\right) \\
e^{-i \frac{\pi}{4}} e^{-i \theta} \sqrt{\mu-I_{e x}} J_{1}\left(\frac{\sqrt{\mu^{2}-I_{e x}^{2}}}{\hbar v} r\right) \\
-e^{i \frac{\pi}{4}} \sqrt{\mu+I_{e x}} J_{0}\left(\frac{\sqrt{\mu^{2}-I_{e x}^{2}}}{\hbar v} r\right)
\end{array}\right],
$$

where $J_{l}$ is the $l$-th Bessel function of the first type, and it is reduced to the Fu-Kane vortex zero-energy mode by setting first $I_{e x}=0$ and then $\mu=0$ [3]. It can also be generalized to $\left|I_{e x}\right|>|\mu|$ using $J_{l}(i x)=i^{n} I_{l}(x)$ for $x \in \mathbb{R}$, where $I_{l}$ is the $l$-th modified Bessel function of the first type, provided, however, that $|\Delta(r \rightarrow \infty)|>\sqrt{I_{e x}^{2}-\mu^{2}}$, i.e., $\left|I_{e x}\right|<\left|I_{e x, c}\right|$, as can be seen from the asymptotic forms for the large real arguments, $I_{l}(x) \sim e^{x} / \sqrt{2 \pi x}$.

On the other hand, when $\left|I_{e x}\right|>\left|I_{e x, c}\right|$, hQAH dominates with the absence of vortex core MZMs. The domain wall CMM can be demonstrated by setting $\mu=0$ with the domain wall at $y=0$ arising from $I_{e x}(y)=I_{0} \Theta(y)$ and $\Delta=\Delta_{0} \Theta(-y)$ 

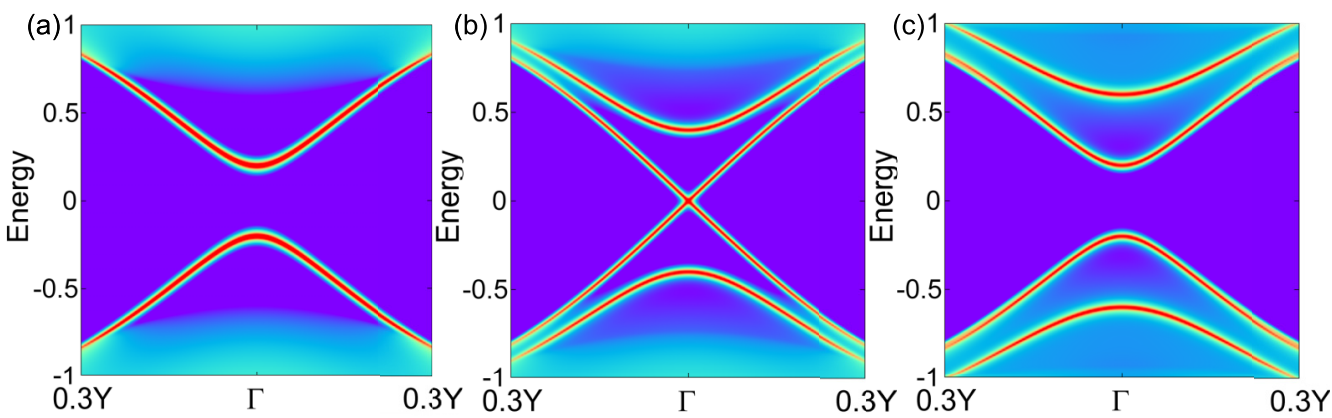

FIG. 2. Topological phase transition for (001) surface states in the lattice model with increasing exchange field $I_{e x}$ : (a) $I_{e x}=0$, (b) $I_{e x}=$ -0.2 and (c) $I_{e x}=-0.4$. The adopted parameters are $t_{x, y}=t_{z}=0.5, M_{0}=2.5, m_{x}=-m_{z}=-1.0, \Delta_{s}=0.2$ and $\mu=0$.

will be considered, i.e.,

$$
\mathcal{H}_{\mathrm{BdG}}=v \mathbf{k} \cdot \boldsymbol{\sigma} \tau_{z}-I_{0} \Theta(y) \sigma_{z}+\Delta_{0} \Theta(-y) \tau_{x}
$$

for $I_{0}=\Delta_{0}>0$, it is straightforward to show the existence of the domain wall CMM,

$$
\left[\begin{array}{c}
\psi_{\uparrow}(\mathbf{r}) \\
\psi_{\downarrow}(\mathbf{r}) \\
\psi_{\downarrow}^{\dagger}(\mathbf{r}) \\
-\psi_{\uparrow}^{\dagger}(\mathbf{r})
\end{array}\right]=\sqrt{\frac{\Delta_{0}}{v L_{x}}} e^{i k_{x} x} e^{-\Delta_{0}|y| / v}\left[\begin{array}{c}
1 / 2 \\
1 / 2 \\
1 / 2 \\
-1 / 2
\end{array}\right]
$$

with the eigenenergy $E_{k_{x}}=v k_{x}$.

\section{MICROSCOPIC MODEL}

Next we will support our results by the numerical simulations based on the bulk model of FeSeTe system. For the FeSeTe bulk system, the topological phase is attributed to a band inversion between two states with opposite parities at $\mathrm{Z}$ point. Taking $\left|S^{+},+\frac{1}{2}\right\rangle,\left|S^{+},-\frac{1}{2}\right\rangle,\left|P^{-}, \frac{1}{2}\right\rangle$, and $\left|P^{-},-\frac{1}{2}\right\rangle$ as the basis at $\mathrm{Z}$ point $[14,15]$, the topological electronic structure can be described by the Hamiltonian in a three-dimensional (3D) lattice $H_{\mathrm{TI}}=\sum_{\boldsymbol{k}} \psi_{\boldsymbol{k}}^{\dagger} \mathcal{H}_{\mathrm{TI}}(\boldsymbol{k}) \psi_{\boldsymbol{k}}$ and the Hamiltonian matrix reads

$$
\mathcal{H}_{\mathrm{TI}}(\boldsymbol{k})=\eta_{x} \boldsymbol{d} \cdot \boldsymbol{\sigma}+M_{\boldsymbol{k}} \eta_{z}-\mu,
$$

where $\psi_{\boldsymbol{k}}^{\dagger}=\left(c_{S \boldsymbol{k} \uparrow}^{\dagger}, c_{S \boldsymbol{k} \downarrow}^{\dagger}, c_{P \boldsymbol{k} \uparrow}^{\dagger}, c_{P \boldsymbol{k} \downarrow}^{\dagger}\right), M_{\mathbf{k}}=M_{0}+m_{z} \cos k_{z}+$ $m_{x}\left(\cos k_{x}+\cos k_{y}\right)$ and $d_{i}=2 t_{i} \sin k_{i}(i=x, y, z)$. Here $\eta$ are Pauli matrices in the orbital space. The mass terms at $\Gamma$ and $Z$ points are $M_{0}+m_{z}+2 m_{x}$ and $M_{0}-m_{z}+2 m_{x}$, respectively. Let us take $m_{0}=-m_{z}=m_{x}$, where the above model describes a strong topological insulator phase with a band inversion at $Z$ point provided $-3<\frac{M_{0}}{m_{0}}<-1$ is satisfied.

We further extend the above Hamiltonian to include superconductivity and an exchange field from impurities. The BdG Hamiltonian is $H_{\mathrm{BdG}}=\frac{1}{2} \sum_{\boldsymbol{k}} \Psi_{\boldsymbol{k}}^{\dagger} \mathcal{H}_{\mathrm{BdG}}^{\mathrm{TI}}(\boldsymbol{k}) \Psi_{\boldsymbol{k}}$ with $\Psi_{\boldsymbol{k}}=$ $\left[\psi_{k}^{\dagger}, \psi_{-k}^{T}\left(-i \sigma_{y}\right)\right]$ and the Hamiltonian matrix reads

$$
\mathcal{H}_{\mathrm{BdG}}^{\mathrm{TI}}(\mathbf{k})=\left(\begin{array}{cc}
\mathcal{H}_{\mathrm{TI}}(\boldsymbol{k})-I_{e x} \sigma_{z} & \Delta_{s} \\
\Delta_{s}^{\dagger} & -\mathcal{H}_{\mathrm{TI}}(\boldsymbol{k})-I_{e x} \sigma_{z}
\end{array}\right),
$$

where $\Delta_{S}$ is the intraorbital spin singlet pairing. In the absence of the exchange field, the (001) surface states will be gapped by superconductivity and form an effective $p+i p$ pairing, where the Majorana modes can be trapped in a vortex core of the surface (as described by Eq. (1) with $I_{e x}=0$ ). We then study the effect of the exchange field on the (001) surface states by adopting the above Hamiltonian with open boundary condition along the $z$ direction.

The microscopic model reproduces the topological phase transition of the low-energy effective theory. Figure 2 demonstrates the process of the topological phase transition for the surface states by fixing the pairing potential and increasing the exchange field strength. In Fig. 2(a), with zero exchange field, the surface state is gapped by the superconducting pairing. When the exchange field strength reaches the critical strength, which is equal to the superconducting gap for $\mu=0$, the gap of the surface states closes [Fig. 2(b)], consistent with the condition of Eq. (2). With further increasing the exchange field, the surface state gap reopens and the system is driven into the hQAH state [Fig. 2(c)].

Next we turn to how the exchange field affects the vortex core MZM in topological surface state superconductivity. We introduce a vortex located at the center of the system by setting $\Delta_{s}(r)=\left|\Delta_{s}(r)\right| e^{i \theta}$ and adopt the Hamiltonian with open boundary conditions along the $x, y, z$ directions. A lattice size of $17 \times 17 \times 16$ is chosen for the following numerical calculations. The exchange field is only restricted to the top (001) surface of the system.

With the above sample configuration, Figs. 3(a) and 3(b) show the distribution of the zero-energy local density of states on the bottom and top surfaces, respectively, for the exchange field exceeding the critical strength defined by Eq. (2). One can see that an "empty vortex" appears on the top surface in Fig. 3(b), in sharp contrast to the "full vortex" on the bottom
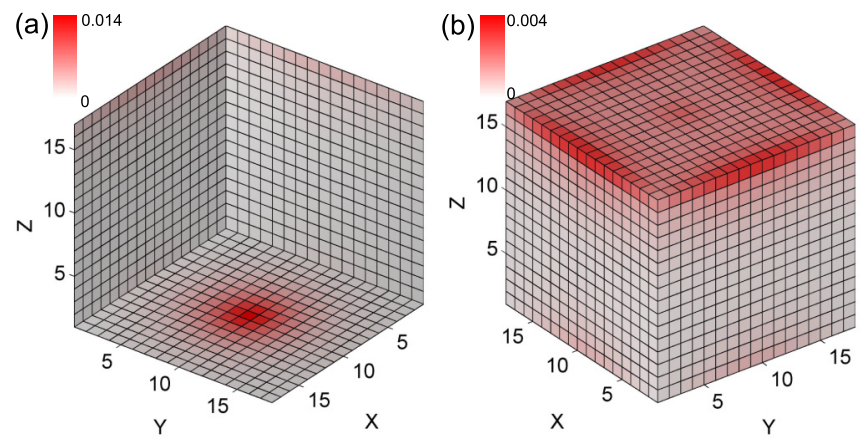

FIG. 3. The 3D profiles of MZMs for (001) surface on a $17 \times$ $17 \times 16$ lattice with $I_{e x}=-0.4, \Delta_{s}=0.2$ and $\mu=0$. There is a localized Majorana in the vortex and chiral Majorana modes localized on edges on bottom and top surface, respectively. 
(a)
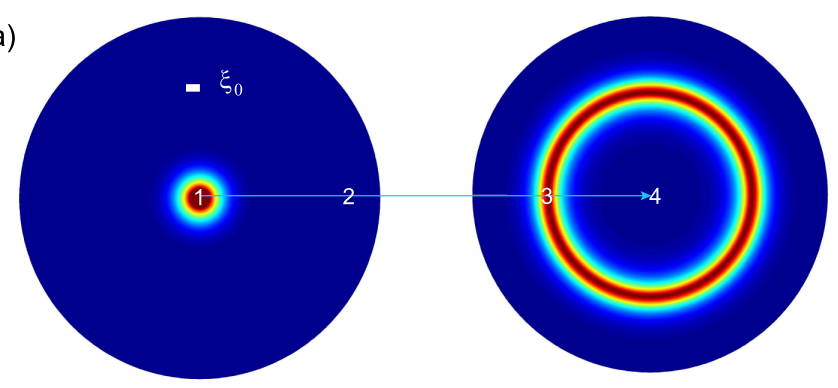

(b)

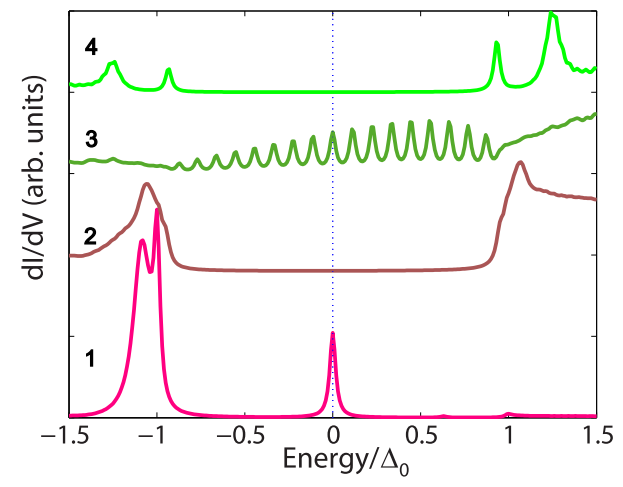

FIG. 4. The profiles of zero-energy states in the vicinity of a "full" vortex and the CMM in an "empty" vortex on the (001) surface (a) and position-dependent local density of states between a "full" and an "empty" vortices (b). $\xi_{0}$ is the coherence length.

surface where there is no exchange field in Fig. 3(a). At the core of a full vortex, there is a well-defined MZM with a zero-bias peak in the local density of states. On the other hand, the MZM is absent at the core of an empty vortex. Despite the absence of MZM in the vortex core, the edges of the top surface under exchange field show a large amount of density of states that depict the presence of edge CMM. In the Appendix A, we study the profile evolution of zero-energy local density of states on the top surface with increasing magnetic fields, from which one can find that the localized MZM gradually extends outside of the vortex and becomes localized on the the edges of (001) surface.

\section{EXPERIMENTAL PREDICTION}

According to angle resolved photoelectron spectroscopy measurements of $\mathrm{Fe}\left(\mathrm{Te}_{0.55} \mathrm{Se}_{0.45}\right)$, the superconducting gap for the surface states is about $\Delta=1.5 \mathrm{meV}$ and the chemical potential (relative to the Dirac point) is about $\mu=$ $4.5 \mathrm{meV}$ [22], resulting in a critical $I_{e x, c}=4.7 \mathrm{meV}$. Our firstprinciples calculation reveals that the average Zeeman field for the surface states from an interstitial Fe impurity is of the order of $10 \mathrm{meV}$ (details are given in the Appendix C). This suggests that interstitial $\mathrm{Fe}$ impurities in $\mathrm{Fe}(\mathrm{Te}, \mathrm{Se})$ can create hQAH regions.

Based on our results that have been well established by both the effective theory and microscopic bulk model, a natural prediction is the existence of the domain wall CMM between an empty vortex and a full vortex. Consider an experimental setup shown in Fig. 1(c), in which two vortices are located at the TSC and hQAH regions, respectively. Figure 4(a) displays the spatial profiles of zero-energy states in the vicinity of a full vortex (left) and an empty vortex (right) and Fig. 4(b) shows the progression of the local density of states (LDOS) as a tip marches from a full vortex to an empty vortex. Experimentally, one can implement an STM measurement of LDOS along the line connecting a full vortex (indicating the TSC state) and an empty vortex (indicating the hQAH state). As shown in Fig. 4(b), a zero-bias peak is expected to occur in the intermediate region without any vortex and can be attributed to the existence of the CMM at the domain wall between the hQAH and TSC regions. This chiral Majorana mode always possessing a zero-energy state is distinct from a normal chiral mode and the energy spectrum is related to the circumference of the region with Zeeman fields (see the Appendix B). With a large thermal smearing in STM measurements, the LDOS at the domain wall exhibits a broad peak around zero energy. While the external magnetic field cannot gap out the CMM, changing its magnitude will shift the location of the CMM as the hQAH region expands while the TSC region contracts or vice versa. The Zeeman gap dominates in vortices of the hQAH regions with only including surface states [Fig. 4(b)]. As there are additional bulk bands in $\mathrm{FeSeTe}$, normal vortex-core states can still appear in such vortices.

\section{CONCLUSION}

To summarize, we reveal that interstitial Fe impurities can create hQAH regions on the surface of FeSeTe, where vortex MZMs are absent but CMM will occur at the boundary. With increasing magnetic field, there will be increasing $\mathrm{Fe}$ impurities with magnetic moments being aligned with the external field. It will enlarge the size of magnetic domains and increase the number of magnetic domains (hQAH). Therefore, our theory naturally explains the experimental observation that zero-bias peaks in some vortices are absent at a weak magnetic field and the fraction of "full" vortices decreases with increasing magnetic fields $[27,28]$. Our mechanism is purely local, i.e., a vortex is "empty" because of its intersecting the surface inside the hQAH domain rather than the long-range MZM interaction effects. It has been known that there should be the CMM localized at the domain wall between regions with dominant superconducting gap and regions with dominant exchange gap. Through an explicit calculation on a minimalistic lattice model of topological bands, we showed that MZM in the vortex core of topological superconductor transforms into the domain wall CMM upon an increase in the exchange field on the region supporting the vortex.

Our proposal is distinct from an earlier proposal in Ref. [29] that relies on pair-wise extinction of MZMs through tunneling between vortices. In our proposal, the MZM relocates and extends to the domain wall CMM instead of disappearing. A clear signature of the proposed mechanism will be the existence of the domain wall CMM between an "empty" vortex and a "full" vortex, which can be detected through STM measurements along a line connecting an "empty" vortex to a nearby "full" vortex. Note that the proposed CMM is distinct from the recent report on the evidence of 1D dispersing helical Majorana modes at the twin boundaries of $\mathrm{Fe}\left(\mathrm{Te}_{0.55} \mathrm{Se}_{0.45}\right)$ [21], which is attributed to the 

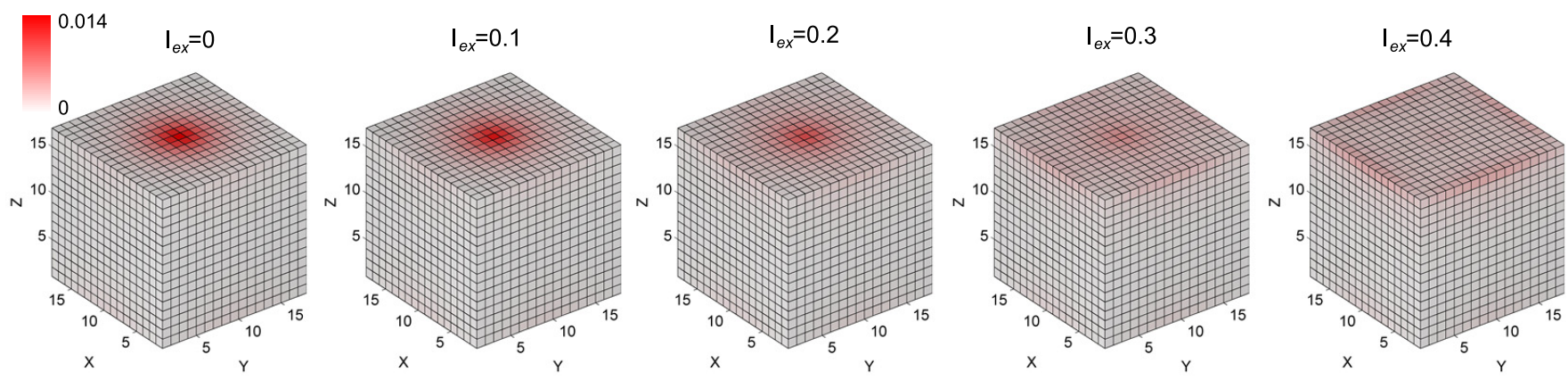

FIG. 5. The profiles of Majorana mode for top (001) surface as a function of $I_{e x}$ (on a $17 \times 17 \times 16$ lattice). $\Delta_{0}=0.2$ and $\mu=0$ are adopted.

$\pi$ junction formed across the crystalline domain wall. Given the clear distinction between the domain wall and the vortex core as shown in Fig. 4(b), our proposal suggests that the CMM detection in Fe $\left(\mathrm{Te}_{0.55} \mathrm{Se}_{0.45}\right)$ through the STM measurement may be relatively easy compared to the recent transport experiments [51,52]. Another prediction that should be easy to check is that we anticipate the "full" vortices and "empty" vortices to segregate as their segregation will represent the regions dominated by TSC or by hQAH.

\section{ACKNOWLEDGMENTS}

We thank H.-H. Wen, T. Hanaguri, and V. Madhavan for useful discussions. E.-A.K. was supported by the National Science Foundation [Platform for the Accelerated Realization, Analysis, and Discovery of Interface Materials (PARADIM)] under Cooperative Agreement No. DMR-1539918. C.L. acknowledges the support of the Office of Naval Research (Grant No. N00014-18-1-2793) and Kaufman New Initiative research Grant No. KA2018-98553 of the Pittsburgh Foundation. S.B.C. was supported by the National Research Foundation of Korea (NRF) grants funded by the Korea government (MSIT) (No. 2020R1A2C1007554) and the Ministry of Education (2018R1A6A1A06024977).

\section{APPENDIX A: EVOLUTION OF MAJORANA MODES ON TOP (001) SURFACE}

We include a Zeeman field on the (001) surface to investigate its effect the on Majorana states. With increasing magnetic field, a topological phase transition on (001) surface states will occur, as shown in Fig. 3 in the main text. If the magnetic field is large enough (larger than $\sqrt{\Delta_{0}^{2}+\mu^{2}}$ ), the (001) surface becomes topologically trivial. As the other sides surface states are topologically nontrivial, chiral Majorana modes should occur. Figure 5 shows the profiles of Majorana modes as a function of Zeeman field $I_{e x}$. With increasing Zeeman field, the localized Majorana mode at vortex core gradually becomes extended and finally transforms into a chiral Majorana mode (on a $17 \times 17 \times 16$ lattice), as demonstrated in Fig. 5.

\section{APPENDIX B: VORTEX STATES IN THE SUPERCONUTING 2D DIRAC SURFACE STATES}

Now we consider the Dirac surface states on the surface of a topological insulator in proximity to a superconductor with an exchange field $B$. The corresponding BdG Hamiltonian reads

$$
H_{s}=v_{F} \tau_{z}\left(p_{x} \sigma_{x}+p_{y} \sigma_{y}\right)-\mu \tau_{z}+B \sigma_{z}+\Delta(r, \theta) \tau_{x}=\left(\begin{array}{cccc}
-\mu+B & p_{x}-i p_{y} & \Delta(r, \theta) & 0 \\
p_{x}+i p_{y} & -\mu-B & 0 & \Delta(r, \theta) \\
\Delta^{\dagger}(r, \theta) & 0 & \mu+B & -p_{x}+i p_{y} \\
0 & \Delta^{\dagger}(r, \theta) & -p_{x}-i p_{y} & \mu-B
\end{array}\right),
$$

where the basis is $\Psi_{\boldsymbol{p}}=\left(c_{\boldsymbol{p} \uparrow}^{\dagger}, c_{\boldsymbol{p} \downarrow}^{\dagger}, c_{-\boldsymbol{p} \downarrow},-c_{-\boldsymbol{p} \uparrow}\right)$. Here $\boldsymbol{\tau}$ and $\boldsymbol{\sigma}$ are Pauli matrices in Nambu and spin space and the gap function $\Delta(r, \theta)=\Delta_{0} f(r) e^{i n \theta}=\Delta_{0} \tanh \frac{r}{\xi_{0}} e^{i n \theta}$ ( $n$ is the vorticity of the vortex). With the above basis, the time reversal operation is $\mathcal{T}=$ $-i \sigma_{y} \mathcal{K}$ and the particle-hole operation $\mathcal{C}=\tau_{y} \sigma_{y} \mathcal{K}$. The above Hamiltonian satisfies $\mathcal{T} H_{s}(\boldsymbol{p}) \mathcal{T}^{-1}=H_{s}(-\boldsymbol{p})$ and $\mathcal{C} H_{s}(\boldsymbol{p}) \mathcal{C}^{-1}=$ $-H_{s}(-\boldsymbol{p})$. In the real space, we use the substitution $p_{x, y} \rightarrow-i \partial_{x, y}$ and we have the following equations:

$$
\begin{gathered}
p_{x}-i p_{y}=-i\left(\partial_{x}-i \partial_{y}\right)=-i e^{-i \theta} \partial_{r}-\frac{e^{-i \theta}}{r} \partial_{\theta}, \\
p_{x}+i p_{y}=-i\left(\partial_{x}+i \partial_{y}\right)=-i e^{i \theta} \partial_{r}+\frac{e^{i \theta}}{r} \partial_{\theta} .
\end{gathered}
$$

As there is a rotational symmetry, the angular momentum is conserved and we can express the above BdG equations as a set of $1 \mathrm{D}$ radial equations separated into angular momentum modes. In the following we consider the vortex with $n=1$ and assume 
the trial wave function has the following form:

$$
\Psi(r, \theta)=\frac{e^{i \nu \theta}}{\sqrt{r}}\left(\begin{array}{c}
e^{i \frac{\pi}{4}} u_{\uparrow}(r) \\
e^{i \theta-i \frac{\pi}{4}} u_{\downarrow}(r) \\
e^{-i \theta+i \frac{\pi}{4}} v_{\downarrow}(r) \\
e^{-i \frac{\pi}{4}} v_{\uparrow}(r)
\end{array}\right)=\frac{e^{i \nu \theta-\frac{i \theta}{2} \sigma_{z}+i \frac{\pi}{4} \sigma_{z}+\frac{i \theta}{2} \tau_{z}}}{\sqrt{r}}\left(\begin{array}{c}
u_{\uparrow}(r) \\
u_{\downarrow}(r) \\
v_{\downarrow}(r) \\
v_{\uparrow}(r)
\end{array}\right)
$$

With the above trial wave function, the eigenequation is $H_{s}\left(r, \theta, \partial_{r}, \partial_{\theta}\right) \Psi(r, \theta)=E \Psi(r, \theta)$ and the matrix form reads

$$
\left(\begin{array}{cccc}
-\mu+B & P_{-} & \Delta(r, \theta) & 0 \\
P_{+} & -\mu-B & 0 & \Delta(r, \theta) \\
\Delta^{\dagger}(r, \theta) & 0 & \mu+B & -P_{-} \\
0 & \Delta^{\dagger}(r, \theta) & -P_{+} & \mu-B
\end{array}\right) \frac{e^{i v \theta}}{\sqrt{r}}\left(\begin{array}{c}
e^{i \frac{\pi}{4}} u_{\uparrow}(r) \\
e^{i \theta-i \frac{\pi}{4}} u_{\downarrow}(r) \\
e^{-i \theta+i \frac{\pi}{4}} v_{\downarrow}(r) \\
e^{-i \frac{\pi}{4}} v_{\uparrow}(r)
\end{array}\right)=E \frac{e^{i v \theta}}{\sqrt{r}}\left(\begin{array}{c}
e^{i \frac{\pi}{4}} u_{\uparrow}(r) \\
e^{i \theta-i \frac{\pi}{4}} u_{\downarrow}(r) \\
e^{-i \theta+i \frac{\pi}{4}} v_{\downarrow}(r) \\
e^{-i \frac{\pi}{4}} v_{\uparrow}(r)
\end{array}\right)
$$

where $P_{-}=-i e^{-i \theta} \partial_{r}-\frac{e^{-i \theta}}{r} \partial_{\theta}$ and $P_{+}=-i e^{i \theta} \partial_{r}+\frac{e^{i \theta}}{r} \partial_{\theta}$. From the above eigenvalue equation, we can get

$$
\begin{gathered}
(-\mu+B) \frac{u_{\uparrow}}{\sqrt{r}}+v_{F}\left(-\partial_{r}-\frac{v+1}{r}\right) \frac{u_{\downarrow}}{\sqrt{r}}+\Delta_{0} f(r) \frac{v_{\downarrow}}{\sqrt{r}}=E \frac{u_{\uparrow}}{\sqrt{r}}, \\
v_{F}\left(\partial_{r}-\frac{v}{r}\right) \frac{u_{\uparrow}}{\sqrt{r}}-(\mu+B) \frac{u_{\downarrow}}{\sqrt{r}}+\Delta_{0} f(r) \frac{v_{\uparrow}}{\sqrt{r}}=E \frac{u_{\downarrow}}{\sqrt{r}}, \\
\Delta_{0} f(r) \frac{u_{\uparrow}}{\sqrt{r}}+(\mu+B) \frac{v_{\downarrow}}{\sqrt{r}}+v_{F}\left(\partial_{r}+\frac{v}{r}\right) \frac{v_{\uparrow}}{\sqrt{r}}=E \frac{v_{\downarrow}}{\sqrt{r}}, \\
\Delta_{0} f(r) \frac{u_{\downarrow}}{\sqrt{r}}-v_{F}\left(\partial_{r}-\frac{v-1}{r}\right) \frac{v_{\downarrow}}{\sqrt{r}}+(\mu-B) \frac{v_{\uparrow}}{\sqrt{r}}=E \frac{v_{\uparrow}}{\sqrt{r}} .
\end{gathered}
$$

Now the radial equations can be further written as

$$
\left(\begin{array}{cccc}
-\mu+B & v_{F}\left(-\partial_{r}-\frac{v+1}{r}\right) & \Delta_{0} f(r) & 0 \\
v_{F}\left(\partial_{r}-\frac{v}{r}\right) & -\mu-B & 0 & \Delta_{0} f(r) \\
\Delta_{0} f(r) & 0 & \mu+B & v_{F}\left(\partial_{r}+\frac{v}{r}\right) \\
0 & \Delta_{0} f(r) & -v_{F}\left(\partial_{r}-\frac{\nu-1}{r}\right) & \mu-B
\end{array}\right) \frac{1}{\sqrt{r}}\left(\begin{array}{l}
u_{\uparrow}(r) \\
u_{\downarrow}(r) \\
v_{\downarrow}(r) \\
v_{\uparrow}(r)
\end{array}\right)=E \frac{1}{\sqrt{r}}\left(\begin{array}{l}
u_{\uparrow}(r) \\
u_{\downarrow}(r) \\
v_{\downarrow}(r) \\
v_{\uparrow}(r)
\end{array}\right) .
$$

Here we notice that the Hamiltonian matrix is not symmetric. For a Majorana state, its antiparticle is itself and the corresponding wave function should satisfy $\mathcal{C} \Psi \propto \Psi$, which leads to $v=0$.

We define $\rho=\frac{r}{\xi_{0}}=\frac{r}{\hbar v_{F} / \Delta_{0}}$ and follow the above definition by setting $\hbar=1$ and we further have $\frac{d}{d r}=\frac{\Delta_{0}}{v_{F}} \frac{d}{d \rho}$ and $\frac{d}{d r} \frac{h}{\sqrt{r}}=$ $\frac{\partial_{r} h}{\sqrt{r}}-\frac{1}{2 r} \frac{h}{\sqrt{r}}$. Therefore, the eigenfunction can be further written as

$$
\left(\begin{array}{cccc}
-\bar{\mu}+\bar{B} & -\partial_{\rho}-\frac{\nu+\frac{1}{2}}{\rho} & f(\rho) & 0 \\
\partial_{\rho}-\frac{\nu+\frac{1}{2}}{\rho} & -\bar{\mu}-\bar{B} & 0 & f(\rho) \\
f(\rho) & 0 & \bar{\mu}+\bar{B} & \partial_{\rho}+\frac{\nu-\frac{1}{2}}{\rho} \\
0 & f(\rho) & -\partial_{\rho}+\frac{\nu-\frac{1}{2}}{\rho} & \bar{\mu}-\bar{B}
\end{array}\right)\left(\begin{array}{l}
u_{\uparrow}(\rho) \\
u_{\downarrow}(\rho) \\
v_{\downarrow}(\rho) \\
v_{\uparrow}(\rho)
\end{array}\right)=\bar{E}\left(\begin{array}{l}
u_{\uparrow}(\rho) \\
u_{\downarrow}(\rho) \\
v_{\downarrow}(\rho) \\
v_{\uparrow}(\rho)
\end{array}\right),
$$

with $\bar{\mu}=\mu / \Delta_{0}, \bar{B}=B / \Delta_{0}$ and $\bar{E}=E / \Delta_{0}$.

When discretizing a Dirac equation on a lattice one encounters the problem of fermion doubling. One standard approach is to use a forward-backward difference scheme for approximating the partial derivatives in the above equations [53-55]:

$$
\begin{array}{ll}
\partial_{\rho} u_{\downarrow} \approx \frac{u_{\downarrow}(\rho+h)-u_{\downarrow}(\rho)}{h} & \left(u_{\downarrow} \rightarrow v_{\downarrow}\right), \\
\partial_{\rho} u_{\uparrow} \approx \frac{u_{\uparrow}(\rho)-u_{\uparrow}(\rho-h)}{h} & \left(u_{\uparrow} \rightarrow v_{\uparrow}\right),
\end{array}
$$


with $h$ being the discretization step. Here we use the same differential form for $u_{\sigma}$ and $v_{\sigma}$ to preserve the particle-hole symmetry. With discretization on 1D radial geometry with radius $R$, the above equation can be written as

$$
\begin{aligned}
& \left(\begin{array}{cccccccc}
-\bar{\mu}+\bar{B} & \frac{1}{h}-\frac{\nu+\frac{1}{2}}{\rho_{j}} & f\left(\rho_{j}\right) & 0 & 0 & -\frac{1}{h} & 0 & 0 \cdots \\
\frac{1}{h}-\frac{\nu+\frac{1}{2}}{\rho_{j}} & -\bar{\mu}-\bar{B} & 0 & f\left(\rho_{j}\right) & 0 & 0 & 0 & 0 \ldots \\
f\left(\rho_{j}\right) & 0 & \bar{\mu}+\bar{B} & \frac{1}{h}+\frac{\nu-\frac{1}{2}}{\rho_{j}} & 0 & 0 & 0 & 0 \ldots \\
0 & f\left(\rho_{j}\right) & \frac{1}{h}+\frac{\nu-\frac{1}{2}}{\rho_{j}} & \bar{\mu}-\bar{B} & 0 & 0 & -\frac{1}{h} & 0 \ldots \\
0 & 0 & 0 & 0 & -\bar{\mu}+\bar{B} & \frac{1}{h}-\frac{v+\frac{1}{2}}{\rho_{j+1}} & f\left(\rho_{j+1}\right) & 0 \ldots \\
-\frac{1}{h} & 0 & 0 & 0 & \frac{1}{h}-\frac{v+\frac{1}{2}}{\rho_{j+1}} & -\bar{\mu}-\bar{B} & 0 & f\left(\rho_{j+1}\right) \ldots \\
0 & 0 & 0 & -\frac{1}{h} & f\left(\rho_{j+1}\right) & 0 & \bar{\mu}+\bar{B} & \frac{1}{h}+\frac{\nu-\frac{1}{2}}{\rho_{j+1}} \ldots \\
0 & 0 & 0 & 0 & 0 & f\left(\rho_{j+1}\right) & \frac{1}{h}+\frac{\nu-\frac{1}{2}}{\rho_{j+1}} & \bar{\mu}-\bar{B} \ldots \\
\vdots & \vdots & \vdots & \vdots & \vdots & \vdots & \vdots & \vdots
\end{array}\right)\left(\begin{array}{c}
u_{\uparrow}\left(\rho_{j}\right) \\
u_{\downarrow}\left(\rho_{j}\right) \\
v_{\downarrow}\left(\rho_{j}\right) \\
v_{\uparrow}\left(\rho_{j}\right) \\
u_{\uparrow}\left(\rho_{j+1}\right) \\
u_{\downarrow}\left(\rho_{j+1}\right) \\
v_{\downarrow}\left(\rho_{j+1}\right) \\
v_{\uparrow}\left(\rho_{j+1}\right) \\
\vdots
\end{array}\right) \\
& =\bar{E}\left(\begin{array}{c}
u_{\uparrow}\left(\rho_{j}\right) \\
u_{\downarrow}\left(\rho_{j}\right) \\
v_{\downarrow}\left(\rho_{j}\right) \\
v_{\uparrow}\left(\rho_{j}\right) \\
u_{\uparrow}\left(\rho_{j+1}\right) \\
u_{\downarrow}\left(\rho_{j+1}\right) \\
v_{\downarrow}\left(\rho_{j+1}\right) \\
v_{\uparrow}\left(\rho_{j+1}\right) \\
\vdots
\end{array}\right) .
\end{aligned}
$$

The above matrix has a general form as

$$
\begin{gathered}
\left(\begin{array}{ccccc}
H_{00}\left(\rho_{j}\right) & H_{01} & 0 & 0 & \cdots \\
H_{01}^{\dagger} & H_{00}\left(\rho_{j+1}\right) & H_{01} & 0 & \cdots \\
0 & H_{01}^{\dagger} & H_{00}\left(\rho_{j+2}\right) & H_{01} & \cdots \\
\vdots & \vdots & \vdots & \vdots & \vdots
\end{array}\right)\left(\begin{array}{c}
\psi\left(\rho_{j}\right) \\
\psi\left(\rho_{j+1}\right) \\
\psi\left(\rho_{j+2}\right) \\
\vdots
\end{array}\right)=\bar{E}\left(\begin{array}{c}
\psi\left(\rho_{j}\right) \\
\psi\left(\rho_{j+1}\right) \\
\psi\left(\rho_{j+2}\right) \\
\vdots
\end{array}\right), \\
H_{00}\left(\rho_{j}\right)=\left(\begin{array}{cccc}
-\bar{\mu}+\bar{B} & \frac{1}{h}-\frac{\nu+\frac{1}{2}}{\rho_{j}} & f\left(\rho_{j}\right) & 0 \\
\frac{1}{h}-\frac{v+\frac{1}{2}}{\rho_{j}} & -\bar{\mu}-\bar{B} & 0 & f\left(\rho_{j}\right) \\
f\left(\rho_{j}\right) & 0 & \bar{\mu}+\bar{B} & \frac{1}{h}+\frac{\nu-\frac{1}{2}}{\rho_{j}} \\
0 & f\left(\rho_{j}\right) & \frac{1}{h}+\frac{v-\frac{1}{2}}{\rho_{j}} & \bar{\mu}-\bar{B}
\end{array}\right), \\
H_{01}=\left(\begin{array}{cccc}
0 & -\frac{1}{h} & 0 & 0 \\
0 & 0 & 0 & 0 \\
0 & 0 & 0 & 0 \\
0 & 0 & -\frac{1}{h} & 0
\end{array}\right),
\end{gathered}
$$

where $h=\frac{R-R_{\min }}{N-1}$ and $\rho_{j}=R_{\min }+(j-1) h$ with $j=1,2, \ldots, N$. In the calculations, we adopt $R_{\min }=0.01, N=1001$, and $R=50$. For the calculations of CMMs, the Zeeman field is assumed to be

$$
B(r)= \begin{cases}B_{0} & r \leqslant R_{0} \\ 0 & r>R_{0}\end{cases}
$$

with $B_{0}=2.2>\sqrt{\mu^{2}+\Delta_{0}^{2}}$. After solving the eigenvalue equation, we calculate the local density of states (LDOS) to simulate the tunneling conductance measured by STM using,

$$
\frac{d I}{d V}(r, E) \propto \operatorname{LDOS}(r, E)=\frac{1}{r} \sum_{\nu n \sigma}\left[\left|u_{n \sigma}^{v}(r)\right|^{2} \delta\left(E-E_{n}^{v}\right)+\left|v_{n \sigma}^{v}(r)\right|^{2} \delta\left(E+E_{n}^{v}\right)\right] .
$$

The spectrum of a vortex in the superconducting Dirac state is displayed in Fig. 6(a). We discard the artificial CMM localized at the outer boundary of the disk. The pink circles denote the bound states inside the vortex and the zero-energy state is the Majorana mode. With including a Zeeman filed $B$ for $|r| \leqslant R_{0}=5$ region, the spectrum is displayed in Fig. 6(b) 

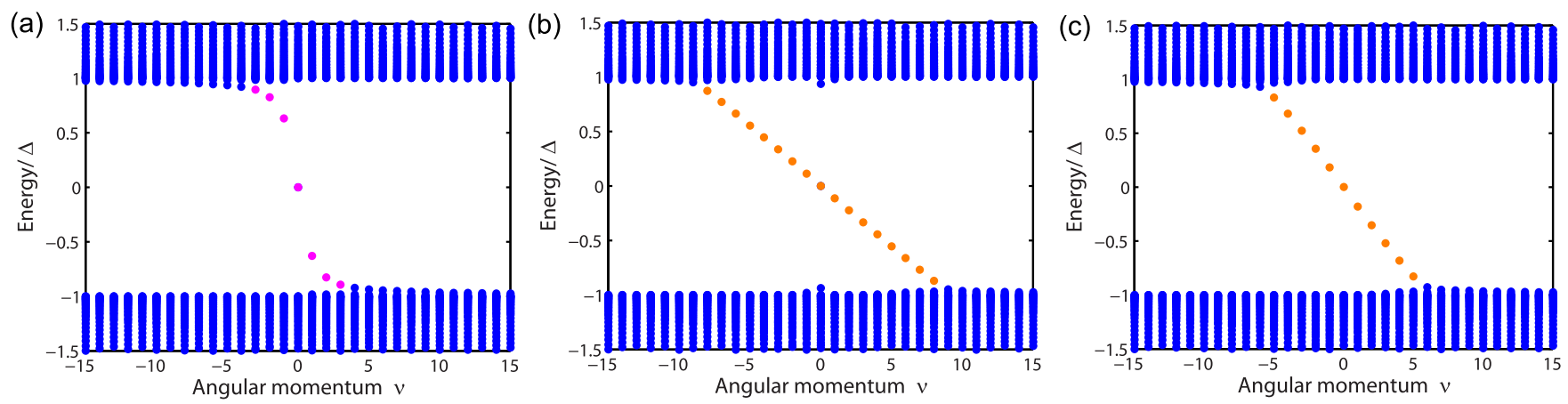

FIG. 6. Energy spectra for a vortex in the superconducting Dirac surface states: (a) $B=0, B(r)=B_{0} \Theta\left(R_{0}-r\right)$ (b) $R_{0}=5$ and (c) $R_{0}=3$. The adopted chemical potential $\mu=1$ and $\Delta(r)=\Delta_{0} \tanh \frac{r}{\xi_{0}}$ with $\Delta_{0}=1$ and $\xi_{0}=1$. The profiles of MZMs as a function of Zeeman field along the $z$ direction.

and the local Majorana mode of the vortex transforms into a CMM (orange circles) localized at the domain wall. The energy quantum of the CMM is proportional to $\frac{1}{L}$ with $L$ being the circumference of the region with exchange field, as shown in Fig. 6(c).

\section{APPENDIX C: ESTIMATION OF AVERAGE ZEEMAN SPLITTING FROM INTERSTITIAL FE IMPURITIES}

About the interstitial iron impurity in $\mathrm{Fe}(\mathrm{Te}, \mathrm{Se})$, neutron scattering measurements suggest that an interstitial Fe can induce a magnetic Friedel-like oscillation and involves $>50$ neighboring Fe sites [38]. Therefore, an interstitial Fe impurity can form a ferromagnetic domain and the magnetic moment of the interstitial Fe is larger than $2 \mu_{B}$. We performed first-principles calculations to estimate the Zeeman splitting from interstitial $\mathrm{Fe}$ impurities. We adopted a $6 \times$ $6 \mathrm{FeTe}_{0.5} \mathrm{Se}_{0.5}$ supercell and put an interstitial Fe impurity in the center. The initial magnetic configuration is taken from
Ref. [38] and is stabilized in the self-consistent calculations, suggesting that an interstitial Fe impurity can indeed induce a magnetic Friedel-like oscillation. The magnetic moment of the interstitial Fe impurity is about $2.5 \mu_{B}$. The magnetic moments of the nearest-neighbor $(\mathrm{NN})$, second $\mathrm{NN}$ and third $\mathrm{NN}$ Fe sites are $1.9,-2.0$ and $2.0 \mu_{B}$, respectively. The Zeeman splitting of $d_{x z / y z}$ orbitals on the NN Fe site is about $200 \mathrm{meV}$. According to our calculations, the average magnetic moment for this cluster is $0.35 \mu_{B}$ per $\mathrm{Fe}$ site. Therefore, the Zeeman splitting parameter for the bulk topological bands $B_{e x}$ is about $18 \mathrm{meV}$. The corresponding Zeeman splitting parameter for the surface states in $\mathrm{Fe}(\mathrm{Te}, \mathrm{Se})$ depends on the details of their wave functions and can be reasonably estimated to be $I_{e x}=\alpha B_{e x}$. Assuming that $\alpha>0.5$, $I_{e x}$ is of the order of $10 \mathrm{meV}$ and this value is greater than the critical value $I_{e x, c}=4.7 \mathrm{meV}$ provided in the main text. Therefore, interstitial Fe impurities in $\mathrm{Fe}(\mathrm{Te}, \mathrm{Se})$ can create magnetic domains and further induce hQAH regions on the surface.
[1] L. Fu and C. L. Kane, Topological insulators with inversion symmetry, Phys. Rev. B 76, 045302 (2007).

[2] X.-L. Qi, T. L. Hughes, and S.-C. Zhang, Topological field theory of time-reversal invariant insulators, Phys. Rev. B 78, 195424 (2008).

[3] L. Fu and C. L. Kane, Superconducting Proximity Effect and Majorana Fermions at the Surface of a Topological Insulator, Phys. Rev. Lett. 100, 096407 (2008).

[4] L. Fu and C. L. Kane, Probing Neutral Majorana Fermion Edge Modes with Charge Transport, Phys. Rev. Lett. 102, 216403 (2009).

[5] M.-X. Wang, C. Liu, J.-P. Xu, F. Yang, L. Miao, M.-Y. Yao, C. L. Gao, C. Shen, X. Ma, X. Chen, Z.-A. Xu, Y. Liu, S.-C. Zhang, D. Qian, J.-F. Jia, and Q.-K. Xue, The coexistence of superconductivity and topological order in the $\mathrm{Bi}_{2} \mathrm{Se}_{3}$ thin films, Science 336, 52 (2012).

[6] M. Veldhorst, M. Snelder, M. Hoek, T. Gang, V. K. Guduru, X. L. Wang, U. Zeitler, W. G. van der Wiel, A. A. Golubov, H. Hilgenkamp, and A. Brinkman, Josephson supercurrent through a topological insulator surface state, Nat. Mater. 11, 417 (2012).
[7] C. Kurter, A. D. K. Finck, Y. S. Hor, and D. J. V. Harlingen, Evidence for an anomalous current-phase relation in topological insulator Josephson junctions, Nat. Commun. 6, 7130 (2015).

[8] J.-P. Xu, M.-X. Wang, Z. L. Liu, J.-F. Ge, X. Yang, C. Liu, Z. A. Xu, D. Guan, C. L. Gao, D. Qian, Y. Liu, Q.-H. Wang, F.-C. Zhang, Q.-K. Xue, and J.-F. Jia, Experimental Detection of a Majorana Mode in the core of a Magnetic Vortex inside a Topological Insulator-Superconductor $\mathrm{Bi}_{2} \mathrm{Te}_{3} / \mathrm{NbSe}_{2}$ Heterostructure, Phys. Rev. Lett. 114, 017001 (2015).

[9] H.-H. Sun, K.-W. Zhang, L.-H. Hu, C. Li, G.-Y. Wang, H.-Y. Ma, Z.-A. Xu, C.-L. Gao, D.-D. Guan, Y.-Y. Li, C. Liu, D. Qian, Y. Zhou, L. Fu, S.-C. Li, F.-C. Zhang, and J.-F. Jia, Majorana Zero Mode Detected with Spin Selective Andreev Reflection in the Vortex of a Topological Superconductor, Phys. Rev. Lett. 116, 257003 (2016).

[10] A. R. Akhmerov, J. Nilsson, and C. W. J. Beenakker, Electrically Detected Interferometry of Majorana Fermions in a Topological Insulator, Phys. Rev. Lett. 102, 216404 (2009).

[11] P. Hosur, P. Ghaemi, R. S. K. Mong, and A. Vishwanath, Majorana Modes at the Ends of Superconductor Vortices in 
Doped Topological Insulators, Phys. Rev. Lett. 107, 097001 (2011).

[12] K. Lee, A. Vaezi, M. H. Fischer, and E.-A. Kim, Superconducting proximity effect in topological metals, Phys. Rev. B 90, 214510 (2014).

[13] N. Hao and J. Hu, Topological Phases in the Single-Layer FeSe, Phys. Rev. X 4, 031053 (2014).

[14] Z. Wang, P. Zhang, G. Xu, L. K. Zeng, H. Miao, X. Xu, T. Qian, H. Weng, P. Richard, A. V. Fedorov, H. Ding, X. Dai, and Z. Fang, Topological nature of the $\mathrm{FeSe}_{0.5} \mathrm{Te}_{0.5}$ superconductor, Phys. Rev. B 92, 115119 (2015).

[15] X. Wu, S. Qin, Y. Liang, H. Fan, and J. Hu, Topological characters in $\mathrm{Fe}\left(\mathrm{Te}_{1-x} \mathrm{Se}_{x}\right)$ thin films, Phys. Rev. B 93, 115129 (2016).

[16] G. Xu, B. Lian, P. Tang, X.-L. Qi, and S.-C. Zhang, Topological Superconductivity on the Surface of Fe-Based Superconductors, Phys. Rev. Lett. 117, 047001 (2016).

[17] R.-X. Zhang, W. S. Cole, and S. Das Sarma, Helical Hinge Majorana Modes in Iron-Based Superconductors, Phys. Rev. Lett. 122, 187001 (2019).

[18] M. J. Gray, J. Freudenstein, S. Y. F. Zhao, R. O'Connor, S. Jenkins, N. Kumar, M. Hoek, A. Kopec, S. Huh, T. Taniguchi, K. Watanabe, R. Zhong, C. Kim, G. D. Gu, and K. S. Burch, Evidence for helical hinge zero modes in an fe-based superconductor, Nano Lett. 19, 4890 (2019).

[19] X. Wu, X. Liu, R. Thomale, and C.-X. Liu, High- $T_{c}$ Superconductor $\mathrm{Fe}(\mathrm{Se}, \mathrm{Te})$ monolayer: an Intrinsic, scalable and electrically-tunable majorana platform, arXiv:1905.10648 (2019).

[20] R.-X. Zhang, W. S. Cole, X. Wu, and S. Das Sarma, HigherOrder Topology and Nodal Topological Superconductivity in $\mathrm{Fe}(\mathrm{Se}, \mathrm{Te})$ Heterostructures, Phys. Rev. Lett. 123, 167001 (2019).

[21] Z. Wang, J. O. Rodriguez, L. Jiao, S. Howard, M. Graham, G. D. Gu, T. L. Hughes, D. K. Morr, and V. Madhavan, Evidence for dispersing 1D Majorana channels in an iron-based superconductor, Science 367, 104 (2020).

[22] P. Zhang, K. Yaji, T. Hashimoto, Y. Ota, T. Kondo, K. Okazaki, Z. Wang, J. Wen, G. D. Gu, H. Ding, and S. Shin, Observation of topological superconductivity on the surface of an iron-based superconductor, Science 360, 182 (2018).

[23] J.-X. Yin, Z. Wu, J.-H. Wang, Z.-Y. Ye, J. Gong, X.-Y. Hou, L. Shan, A. Li, X.-J. Liang, X.-X. Wu, J. Li, C.-S. Ting, Z.-Q. Wang, J-P. Hu, P.-H. Hor, H. Ding, and S. H. Pan, Observation of a robust zero-energy bound state in iron-based superconductor Fe(Te,Se), Nat. Phys. 11, 543 (2015).

[24] D. Wang, L. Kong, P. Fan, H. Chen, S. Zhu, W. Liu, L. Cao, Y. Sun, S. Du, J. Schneeloch, R. Zhong, G. Gu, L. Fu, H. Ding, and H.-J. Gao, Evidence for Majorana bound states in an iron-based superconductor, Science 362, 333 (2018).

[25] L. Kong, S. Zhu, M. Papaj, H. Chen, L. Cao, H. Isobe, Y. Xing, W. Liu, D. Wang, P. Fan, Y. Sun, S. Du, J. Schneeloch, R. Zhong, G. Gu, L. Fu, H.-J. Gao, and H. Ding, Half-integer level shift of vortex bound states in an iron-based superconductor, Nat. Phys. 15, 1181 (2019).

[26] S. Zhu, L. Kong, L. Cao, H. Chen, M. Papaj, S. Du, Y. Xing, W. Liu, D. Wang, C. Shen, F. Yang, J. Schneeloch, R. Zhong, G. Gu, L. Fu, Y.-Y. Zhang, H. Ding, and H.-J. Gao, Nearly quantized conductance plateau of vortex zero mode in an iron-based superconductor, Science 367, 189 (2020).
[27] X. Chen, M. Chen, W. Duan, X. Zhu, H. Yang, and H.-H. Wen, Observation and characterization of the zero energy conductance peak in the vortex core state of $\mathrm{FeTe}_{0.55} \mathrm{Se}_{0.45}$ (2019), arXiv:1909.01686 [cond-mat.supr-con].

[28] T. Machida, Y. Sun, S. Pyon, S. Takeda, Y. Kohsaka, T. Hanaguri, T. Sasagawa, and T. Tamegai, Zero-energy vortex bound state in the superconducting topological surface state of $\mathrm{Fe}(\mathrm{Se}, \mathrm{Te})$, Nat. Mater. 18, 811 (2019).

[29] C.-K. Chiu, T. Machida, Y. Huang, T. Hanaguri, and F.-C. Zhang, Scalable majorana vortex modes in iron-based superconductors, Sci. Adv. 6, eaay0443 (2020).

[30] C.-X. Liu, X.-L. Qi, X. Dai, Z. Fang, and S.-C. Zhang, Quantum Anomalous Hall Effect in $\mathrm{Hg}_{1-y} \mathrm{Mn}_{y}$ Te Quantum Wells, Phys. Rev. Lett. 101, 146802 (2008).

[31] C.-Z. Chang, J. Zhang, X. Feng, J. Shen, Z. Zhang, M. Guo, K. Li, Y. Ou, P. Wei, L.-L. Wang, Z.-Q. Ji, Y. Feng, S. Ji, X. Chen, J. Jia, X. Dai, Z. Fang, S.-C. Zhang, K. He, Y. Wang, L. Lu, X.-C. Ma, and Q.-K. Xue, Experimental observation of the quantum anomalous Hall effect in a magnetic topological insulator, Science 340, 167 (2013).

[32] P. Wei, F. Katmis, B. A. Assaf, H. Steinberg, P. Jarillo-Herrero, D. Heiman, and J. S. Moodera, Exchange-Coupling-Induced Symmetry Breaking in Topological Insulators, Phys. Rev. Lett. 110, 186807 (2013).

[33] P. O. Sprau, A. Kostin, A. Kreisel, A. E. Böhmer, V. Taufour, P. C. Canfield, S. Mukherjee, P. J. Hirschfeld, B. M. Andersen, and J. C. S. Davis, Discovery of orbital-selective Cooper pairing in FeSe, Science 357, 75 (2017).

[34] D. Liu, C. Li, J. Huang, B. Lei, L. Wang, X. Wu, B. Shen, Q. Gao, Y. Zhang, X. Liu, Y. Hu, Y. Xu, A. Liang, J. Liu, P. Ai, L. Zhao, S. He, L. Yu, G. Liu, Y. Mao, X. Dong, X. Jia, F. Zhang, S. Zhang, F. Yang, Z. Wang, Q. Peng, Y. Shi, J. Hu, T. Xiang, X. Chen, Z. Xu, C. Chen, and X. J. Zhou, Orbital Origin of Extremely Anisotropic Superconducting Gap in Nematic Phase of FeSe Superconductor, Phys. Rev. X 8, 031033 (2018).

[35] X.-L. Qi, T. L. Hughes, and S.-C. Zhang, Chiral topological superconductor from the quantum hall state, Phys. Rev. B 82, 184516 (2010).

[36] S. B. Chung, X.-L. Qi, J. Maciejko, and S.-C. Zhang, Conductance and noise signatures of Majorana backscattering, Phys. Rev. B 83, 100512(R) (2011).

[37] J. Wang, Q. Zhou, B. Lian, and S.-C. Zhang, Chiral topological superconductor and half-integer conductance plateau from quantum anomalous Hall plateau transition, Phys. Rev. B 92, 064520 (2015).

[38] V. Thampy, J. Kang, J. A. Rodriguez-Rivera, W. Bao, A. T Savici, J. Hu, T. J. Liu, B. Qian, D. Fobes, Z. Q. Mao, C. B. Fu, W. C. Chen, Q. Ye, R. W. Erwin, T. R. Gentile, Z. Tesanovic, and C. Broholm, Friedel-Like Oscillations from Interstitial Iron in Superconducting $\mathrm{Fe}_{1+y} \mathrm{Te}_{0.62} \mathrm{Se}_{0.38}$, Phys. Rev. Lett. 108, 107002 (2012).

[39] K. Jiang, X. Dai, and Z. Wang, Quantum Anomalous Vortex and Majorana Zero Mode in Iron-Based Superconductor Fe(Te,Se), Phys. Rev. X 9, 011033 (2019).

[40] Q. Liu, C.-X. Liu, C. Xu, X.-L. Qi, and S.-C. Zhang, Magnetic Impurities on the Surface of a Topological Insulator, Phys. Rev. Lett. 102, 156603 (2009).

[41] Y. L. Chen, J.-H. Chu, J. G. Analytis, Z. K. Liu, K. Igarashi, H.-H. Kuo, X. L. Qi, S. K. Mo, R. G. Moore, D. H. Lu, M. Hashimoto, T. Sasagawa, S. C. Zhang, I. R. Fisher, Z. Hussain, 
and Z. X. Shen, Massive Dirac fermion on the surface of a magnetically doped topological insulator, Science 329, 659 (2010).

[42] L. A. Wray, S.-Y. Xu, Y. Xia, D. Hsieh, A. V. Fedorov, Y. S. Hor, R. J. Cava, A. Bansil, H. Lin, and M. Z. Hasan, A topological insulator surface under strong Coulomb, magnetic, and disorder perturbations, Nat. Phys. 7, 32 (2011).

[43] J. Henk, M. Flieger, I. V. Maznichenko, I. Mertig, A. Ernst, S. V. Eremeev, and E. V. Chulkov, Topological Character and Magnetism of the Dirac State in Mn-Doped $\mathrm{Bi}_{2} \mathrm{Te}_{3}$, Phys. Rev. Lett. 109, 076801 (2012).

[44] G. Rosenberg and M. Franz, Surface magnetic ordering in topological insulators with bulk magnetic dopants, Phys. Rev. B 85, 195119 (2012).

[45] D. K. Efimkin and V. Galitski, Self-consistent theory of ferromagnetism on the surface of a topological insulator, Phys. Rev. B 89, 115431 (2014).

[46] A. Ghazaryan, P. L. S. Lopes, P. Hosur, M. J. Gilbert, and P. Ghaemi, Effect of Zeeman coupling on the Majorana vortex modes in iron-based topological superconductors, Phys. Rev. B 101, 020504(R) (2020).

[47] I. Lee, C. K. Kim, J. Lee, S. J. L. Billinge, R. Zhong, J. A. Schneeloch, T. Liu, T. Valla, J. M. Tranquada, G. Gu, and J. C. S. Davis, Imaging Dirac-mass disorder from magnetic dopant atoms in the ferromagnetic topological insulator $\mathrm{Cr}_{x}\left(\mathrm{Bi}_{0.1} \mathrm{Sb}_{0.9}\right)_{2-x} \mathrm{Te}_{3}$, Proc. Natl. Acad. Sci. USA 112, 1316 (2015).
[48] M. Sato, Y. Takahashi, and S. Fujimoto, Non-Abelian Topological Order in $s$-Wave Superfluids of Ultracold Fermionic Atoms, Phys. Rev. Lett. 103, 020401 (2009).

[49] J. D. Sau, R. M. Lutchyn, S. Tewari, and S. D. Sarma, Generic New Platform for Topological Quantum Computation Using Semiconductor Heterostructures, Phys. Rev. Lett. 104, 040502 (2010).

[50] J. Alicea, Majorana fermions in a tunable semiconductor device, Phys. Rev. B 81, 125318 (2010).

[51] Q. L. He, L. Pan, A. L. Stern, E. C. Burks, X. Che, G. Yin, J. Wang, B. Lian, Q. Zhou, E. S. Choi, K. Murata, X. Kou, Z. Chen, T. Nie, Q. Shao, Y. Fan, S.-C. Zhang, K. Liu, J. $\mathrm{Xia}$, and K. L. Wang, Chiral Majorana fermion modes in a quantum anomalous Hall insulator-superconductor structure, Science 357, 294 (2017).

[52] Y. Kasahara, T. Ohnishi, Y. Mizukami, O. Tanaka, S. Ma, K. Sugii, N. Kurita, H. Tanaka, J. Nasu, Y. Motome, T. Shibauchi, and Y. Matsuda, Majorana quantization and half-integer thermal quantum Hall effect in a Kitaev spin liquid, Nature 559, 227 (2018).

[53] L. Susskind, Lattice fermions, Phys. Rev. D 16, 3031 (1977).

[54] R. Stacey, Eliminating lattice fermion doubling, Phys. Rev. D 26, 468 (1982).

[55] C. Gutiérrez, D. Walkup, F. Ghahari, C. Lewandowski, J. F. Rodriguez-Nieva, K. Watanabe, T. Taniguchi, L. S. Levitov, N. B. Zhitenev, and J. A. Stroscio, Interaction-driven quantum Hall wedding cake-like structures in graphene quantum dots, Science 361, 789 (2018). 